"Los vegetales que viven en la República". El estudio de la flora uruguaya en Anales del Museo Nacional de Montevideo, 18941925

\title{
"Los vegetales que viven en la República". E1 estudio de la flora uruguaya en Anales del Museo Nacional de Montevideo, 1894-1925
}

\author{
"The vegetables that live in the Republic." The study of \\ the Uruguayan flora in Anales del Museo Nacional de \\ Montevideo, 1894-1925
}

RODRIGO ANTONIO VEGA Y ORTEGA BAEZ

Universidad Nacional Autónoma de México (México) rodrigo.vegayortega@hotmail.com

RESUMEN

Entre 1894 y 1925, Anales del Museo Nacional de Montevideo publicó veintiséis escritos botánicos de la autoría de diez autores, algunos residentes en el Uruguay y otros en el extranjero. El objetivo del artículo es caracterizar las prácticas botánicas desarrolladas por los autores vinculados con el Museo en cuanto a la elaboración del inventario de la flora uruguaya. Los temas en que se divide el artículo son la colecta botánica en las excursiones; el estudio, descripción y clasificación de las especies en el Museo; la práctica experimental en las instituciones científicas de Montevideo; y la utilidad de algunas plantas uruguayas. Los cuatro temas muestran las prácticas científicas que llevaron a cabo los autores al estudiar la flora del país, así como que la revista fungió como un medio impreso que hace evidente la coproducción de conocimiento científico entre diferentes especialistas en la naturaleza del Uruguay ubicados en varias partes del mundo.

Palabras clave: Uruguay, Botánica, Prensa, Museo, Ciencia

ABSTRACT

Between 1894 and 1925, Anales del Museo Nacional de Montevideo published twentysix botanical writings by ten authors, some residents in Uruguay and others abroad. The objective of the article is to characterize the botanical practices developed by the authors associated with the Museum in terms of the elaboration of the Uruguayan flora inventory. The topics in which the article is divided are the botanical collection in the excursions; the study, description and classification of the species in the Museum; the experimental practice in the scientific institutions of Montevideo; and the usefulness of 
some Uruguayan plants. The four topics show the scientific practices that the authors carried out when studying the flora of the country, as well as that the journal served as a printed medium that makes evident the co-production of scientific knowledge between different specialists in the nature of Uruguay located in various parts of the world.

Keywords: Uruguay, Botany, Press, Museum, Science.

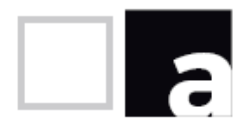

La historia de la ciencia uruguaya ha recibido poca atención por los historiadores a pesar de la amplia tradición que se remonta a la época colonial y que se dinamizó después de la independencia. No obstante, existe una amplia y diversa cantidad de fuentes archivísticas, bibliográficas, hemerográficas, iconográficas, orales y materiales relativas a distintas disciplinas científicas.

Este artículo se encuentra enmarcado en una investigación más amplia sobre la historia de las ciencias naturales que abarca desde la fundación del Estado Oriental del Uruguay en 1828 hasta el inicio del gobierno de Gabriel Terra en 1931. Como un primer estudio de caso se presenta el análisis de la práctica botánica al interior del Museo Nacional de Montevideo a través de su revista y como ejemplo de las actividades fundacionales de la institución.

En efecto, en 1894 inició la publicación de Anales del Museo Nacional de Montevideo (AMNM), primera revista editada por la institución uruguaya, cuyo redactor fundador fue José Arechavaleta (1838-1912), naturalista de origen español radicado en Montevideo, quien a su vez fungía como director del Museo. La revista dio a conocer escritos de zoología, botánica, geología, geografia, mineralogía, arqueología y antropología de autores nacionales y extranjeros. AMNM abarca siete volúmenes de la primera serie (1894-1911) y el primer volumen de la segunda serie de 1925 que incluyó escritos anteriores a 1911.1 En 1926, la revista cambió de denominación a Anales del Museo de Historia Natural de Montevideo.

El objetivo del artículo es caracterizar las prácticas botánicas desarrolladas por los autores de $A M N M$, varios de ellos fueron naturalistas vinculados con el

${ }^{1}$ La investigación abarca los cinco escritos póstumos de Arechavaleta fechados en 1904 y 1905 e incluidos en el volumen de 1925. 
Museo en el periodo 1894-1911. En este lapso, los escritos botánicos incluyeron tanto plantas como hongos, pues estos últimos se estudiaban dentro del reino vegetal. Los autores residentes en el Uruguay fueron José Arechavaleta, ${ }^{2}$ Carlos Regúnaga, ${ }^{3}$ Mariano B. Berro (1833-1919),, ${ }^{4}$ Guillermo Herter (1884-1954)5 y Joseph-Ernest Gibert (1818-1886), ${ }^{6}$ mientras que los botánicos europeos de los que se publicaron traducciones sobre la flora uruguaya fueron Otto Kuntze (1843-1907)7 de Alemania, Édouard-François André (1840-1911)8 de Francia, el capitán Baker ${ }^{8}$ de Gran Bretaña, Carlos Spegazzini (1858-1926) ${ }^{10}$ italiano residente en La Plata y J. Müller ${ }^{11}$ de Suiza.

La fuente histórica se compone de una muestra representativa de veinte escritos relativos a las especies vegetales del Uruguay, de un total de veintiséis. ${ }^{12}$ Los temas en que se divide el artículo son la colecta botánica en las excursiones; el estudio, descripción y clasificación de las especies; la práctica experimental en las instituciones científicas de Montevideo; y la utilidad de algunas plantas. Los cuatro temas muestran las prácticas científicas que llevaron a cabo los autores al estudiar la flora del país, así como que $A M N M$ fungió como un medio impreso que puso en contacto a diferentes especialistas en la naturaleza del Uruguay ubicados en varias partes del mundo. ${ }^{13}$

De acuerdo con Jim Endersby (2008), la botánica al final del siglo XIX tuvo dos líneas generales de investigación: "la clasificación científica del mundo y la apropiación de los recursos naturales" a partir de observar, colectar, describir, clasificar y exhibir la flora de cada país y el mundo en colecciones públicas y

\footnotetext{
2 Radicó en Montevideo desde 1856. Fue profesor de la Facultad de Medicina de la Universidad, director del Museo Nacional y fundador de la Sociedad "El Microscopio".

3 Jefe director del Laboratorio Municipal de Química.

${ }^{4}$ Fue un político y naturalista uruguayo. Se desempeñó como diputado por el Partido Nacional.

5 Botánico de origen alemán radicado en el Uruguay entre 1910 y 1940.

6 Abogado y naturalista francés. Radicó en Montevideo desde 1851.

7 Fue un farmacéutico y botánico alemán que recorrió varias partes del mundo con el propósito de encontrar plantas útiles a la industria. Su obra se compone de 48,727 registros de nuevas especies.

8 Viajero que recorrió varios países sudamericanos con el propósito de colectar especies de la flora.

${ }^{9}$ Hasta ahora no ha sido posible identificar con certeza a este autor.

10 Naturalista italiano egresado de la Real Escuela de Viticultura y Enología en Conegliano.

Desde 1879 radicó en Sudamérica.

11 Sobre este naturalista sólo se ha detectado que radicó en Ginebra.

12 Los temas en relación con el número de escritos en AMNM son: botánica (27), zoología (11), institucional (3), geografia (2), geología (1) y química (1).

13 Los escritos botánicos que no se analizan son seis porque presentan los mismos temas de la muestra representativa. Édouard-François André, "Sobre nomenclatura botánica hortícola" (1899), Otto Kuntze, "Géneros de plantas anteriores al año 1891, reformados legalmente según las reglas que sirvieron a Engler para las familias" (1899), José Arechavaleta, "Notas sobre la obra de Pritzel: Thesaurus Literaturae Botanicae" (1903), José Arechavaleta, "Otras noticias referentes a Pritzel" (1903), Carlos Spegazzini, "Stipeae platenses" (1903) y José Arechavaleta, "Flora Uruguaya" (1911). El único texto publicado en otra lengua, en este caso latín, fue "Stipeae platenses" de Spegazzini.
} 
privadas (p. 18). La botánica fue una ciencia que en el periodo de esta investigación reunió a numerosos practicantes dispersos por el mundo, los cuales se comunicaban a través de correspondencia e impresos (libros y revistas). La sistematización de la información botánica se centralizó en instituciones y agrupaciones en dos niveles: el nacional, en que los museos, jardines botánicos y agrupaciones de naturalistas acopiaron datos y ejemplares de la flora de un país; y el global, en que algunas instancias nacionales tuvieron una vocación imperial al reunir información sobre la flora extranjera, en ocasiones con miras a "la utilidad que tiene para dar a conocer los recursos naturales de tierras lejanas" (Drayton, 2000, p. 72). Los museos científicos fueron instituciones que destinaron amplios recursos al coleccionismo de la naturaleza nacional y a veces mundial, así como dar a conocer los resultados de investigación en libros y revistas, como el caso del museo uruguayo.

La historiografia de la botánica uruguaya es escasa a pesar de la tradición naturalista en el país. Uno de los primeros recuentos históricos sobre los practicantes de la botánica se encuentra en el "Prólogo" escrito por José Arechavaleta (1905b) para el quinto volumen de $A M N M$ en que expuso los nombres y aportaciones de numerosos individuos nacionales y extranjeros desde el periodo colonial hasta inicios del siglo XX. A finales de dicha centuria se publicaron otros estudios generales sobre la dinámica científica del Uruguay, como los de Alción Cheroni (1988) y Fernando Mañé (1996), sin que ambos autores dediquen un estudio particular al Museo Nacional.

En la historiografia también se han elaborado estudios biográficos de algunos de los autores de AMNM. En primer lugar se encuentran distintas biografias del señalado José Arechavaleta escritas por Antonio Peluffo (1989), Ángel Goicoetxea (1993) y Fernando Mañé (2017). Sobre Carlos Spegazzini han escrito Liliana Katinas, Diego G. Gutiérrez y Silvia S. Torres (2000), así como Adriana S. Fiedczuk y Alberto A. De Magistris (2016). Frans A. Stafleu (1978) ha analizado la obra de Otto Kuntze (1843-1907); Héctor A. Aguilar (2009) ha biografiado a Carlos Berg; André Florence y Stéphanie de Courtois (2001) han estudiado la vida de Édouard-François André; el estudio biográfico del emigrante francés José Ernesto Gibert es de la autoría de José María Fernández Saldaña (1945); Walter Rela (2002) se ha centrado en Mariano B. Berro; y Arturo Scarone (1937) dedica una semblanza al naturalista alemán Guillermo Herter. Hasta ahora no se ha encontrado un estudio sobre Carlos Regúnaga. Estos naturalistas fueron actores destacados en el devenir del Museo Nacional y dieron a conocer resultados de investigación en su revista, como se presentará más adelante.

En los últimos años se han publicado varias investigaciones relativas a la historia de la producción agropecuaria del Uruguay en los siglos XIX y XX, la cual requirió de los conocimientos y las prácticas de la ciencia, como han mostrado Alfredo R. Castellanos (1973), Belén Baptista (2005), Daniele Bonfanti 
(2012), María Inés Moraes (2012), Verónica Sanz (2016), Alcides

Beretta (2017), entre otros, quienes han retomado la participación de las instancias científicas uruguayas y las acciones de algunos de los botánicos aquí analizados.

En cuanto a la historia del Museo Nacional resalta el estudio general de Álvaro Mones (2011) y el particular de Miguel A. Klappenbach (2004), aunque hace falta un mayor número de investigaciones pormenorizadas sobre los siglos XIX y XX. ${ }^{14} A M N M$ tampoco ha merecido un estudio profundo como un medio de comunicación en la producción científica uruguaya.

La metodología retoma el papel de las revistas especializadas en la convivencia intelectual de autores con diferentes origenes socioprofesionales, étnicos, institucionales, geográficos, entre otras cuestiones, relacionados con la producción de conocimiento científico en el marco del proceso de comunicar los resultados de investigación como han estudiado James Secord (2004) y Leslie Howsam (2006).

Lynn K. Nyhart (1991) indica que las revistas científicas arroparon la dinámica social de las comunidades especializadas al final del siglo XIX al manifestarse como un instrumento para apuntalar la "comunicación entre especialistas, establecer reclamos para mejorar sus estatus y controlar el acceso a las oportunidades de publicar, así como para obtener reconocimiento y recompensas de la sociedad y el Estado" (p. 43). Para Alex Csiszar (2018), la revista científica ha sido empleada para identificar a los autores como legitimos practicantes de la ciencia y expertos calificados "en los temas relevantes del conocimiento" entre sus pares académicos y los grupos políticos y económicos (p. 3). En este sentido, los naturalistas uruguayos se sumaron a la dinámica hemerográfica especializada que había ganado terreno en la discusión científica internacional. $A M N M$, como otras revistas de instituciones científicas del mundo, fue parte del proceso de conformación de un nuevo estatus académico (Fox, 2016, p. 16).

De acuerdo con Melinda Baldwin (2015), la prensa científica "participó en el establecimiento del hombre de ciencia como categoría intelectual a partir de la elaboración de investigación científica original, ya fuera su aplicación práctica o la creación de conocimiento teórico" (p. 75). Este fenómeno comunicativo se aprecia entre los botánicos de $A M N M$, quienes recibieron apoyo por parte del gobierno nacional con el objetivo de desarrollar ciencia local para inventariar

${ }_{14}$ El Museo uruguayo fue parte de la dinámica museística latinoamericana de finales del siglo XIX, como se aprecia en los museos de Argentina, México, Costa Rica, Brasil, Chile y Colombia, los cuales también publicaron impresos científicos. 
los recursos naturales del país con miras a establecer su control y aprovechamiento (Greiff \& Nieto, 2006, p. 245).

$A M N M$ visibiliza la red de botánicos que radicaban en el Uruguay y en otros países europeos y americanos que estudiaban las especies vegetales del país en el marco del inventario de la flora del mundo y, en particular, la sudamericana. La mayoría de los escritos botánicos muestran el interés de los autores por presentar al público la utilidad de varias especies en términos agrícolas, ganaderos, artesanales, industriales, comerciales y terapéuticos. La revista refleja las asimetrías entre los naturalistas europeos y los uruguayos en cuanto al conocimiento pormenorizado de la flora del país, como científicos viajeros o residentes en América del Sur, así como el contacto entre el Museo Nacional y algunas instituciones europeas y argentinas.

También se retoma el término "coproducción de conocimiento" en que participaron los naturalistas europeos con los científicos uruguayos en el inventario de la flora del país y "alude a las contribuciones de actores locales con diversas habilidades relacionadas" con la exploración de la naturaleza que "fueron cruciales para la puesta en operación" del aprovechamiento de los recursos vegetales (Azuela, 2020, p. 4). AMNM refleja que la experiencia naturalista local resultó imprescindible en la coproducción de artículos, informes, catálogos y traducciones entre autores europeos y uruguayos al momento de determinar la taxonomía de algunas especies. La revista del Museo también hace notar que los naturalistas uruguayos se auxiliaron de una amplia gama de personas provenientes de diversos estratos sociales para reconocer la flora nacional, ya fueran curanderos, guías, ganaderos, terratenientes, artesanos, militares, sacerdotes, entre otros, "que les proporcionaban información y conocimiento" local (Shaffer, Roberts, Raj \& Delbourgo, 2009, p. $\mathrm{XI})$.

Roberts (2009) indica que en la coproducción "la mayor parte de la actividad científica y tecnológica ha implicado históricamente la adaptación y el uso local de los conocimientos, procedimientos y aparatos" (p. 27). En la coproducción de conocimiento, los naturalistas uruguayos mantuvieron contacto epistolar con sus pares europeos, quienes en ocasiones recorrieron el Uruguay y en otras sólo conocieron la flora mediante los envíos de especímenes que se hicieron desde Montevideo. En la revista se encuentran trazos de la circulación de resultados científicos de la coproducción de "conocimientos, técnicas", textos, y representaciones de la naturaleza uruguaya (Roberts, 2009, p. 15).

Kapil Raj (2007) señala que "las proposiciones, los artefactos y las prácticas científicas no son innatamente universales (por su fuerza epistemológica) ni se imponen por la fuerza a otros. Se difunden a través de complejos procesos de asimilación y negociación, tan contingentes como los involucrados en su producción" (p. 9). AMNM evidencia las actividades de una comunidad científica 
en formación, reducida en número, pero dinámica en cuanto al escrutinio de la naturaleza uruguaya, cuyos miembros establecieron amplias relaciones con los naturalistas de las metrópolis científicas del Viejo Mundo y con algunas instituciones científicas de la Argentina.

Los naturalistas uruguayos apoyados por los distintos presidentes en el lapso 1894-1911 se unieron a una serie de políticas científicas orientadas al aprovechamiento racional de los recursos naturales en función del comercio de materias primas rumbo a Europa y el consumo interno. Los científicos estudiaron las especies uruguayas tanto las endémicas como las que se compartían con otros países americanos y del mundo con miras a presentar su utilidad desde los parámetros de la ciencia.

Hay que tener en cuenta que entre 1890 y 1915, el mercado internacional demandó una gran cantidad de materias primas de origen vegetal y animal, para lo cual los gobiernos del Uruguay iniciaron un proyecto de expansión de la producción de materias primas, por lo que "fue necesario introducir y adoptar nuevos conocimientos científicos para que los productores se encontraran en condiciones de competir en los mercados mundiales" (Beretta, 2020, p. 183). A pesar de las revoluciones de 1897 (marzo a septiembre) y 1904 (enero a septiembre), las actividades científicas en el país continuaron y los naturalistas relacionados con el Museo mantuvieron la publicación de los volúmenes de AMNM.

\section{E1 Museo}

Los orígenes del actual Museo Nacional de Historia Natural de Montevideo se encuentran en el decreto de fundación del 4 de septiembre de 1837 a partir de los trabajos de una comisión para organizar la Biblioteca Pública y el Museo de Historia Natural (Mañé, 1996, p. 27). En la década de 1830, el presidente Manuel Oribe dio los primeros pasos para establecer las primeras instituciones científicas y financiar expediciones de reconocimiento territorial.

E1 Museo se inauguró el 18 de julio de 1838 en la llamada Casa del Gobernador, donde se exhibieron objetos naturalistas, geológicos y arqueológicos. Durante varios años la institución tuvo diversas problemáticas para cumplir sus tareas científicas, hasta que en 1868, el gobierno formó una comisión para reformar el Museo, en la cual participó José Arechavaleta. Esta propuso varios cambios para darle un carácter científico. Otro cambio tuvo lugar en 1880, cuando el gobierno dispuso la separación institucional del Museo y la Biblioteca nacionales. A partir de entonces, el Museo Nacional se compuso de las secciones de Historia Natural, Bellas Artes e Historia (Mones, 2011, p. 6). 
El presidente Julio Herrera y Obes se interesó en modernizar las instituciones culturales del país, como se aprecia en marzo de 1890 cuando encargó a Arechavaleta que reorganizara al Museo, "tarea continuada por el Dr. Carlos Berg (1843-1902), quien fue nombrado director en el mes de julio del mismo año" (Mones, 2011, p. 9). La nueva apertura del Museo se llevó a cabo en septiembre de 1891 como una institución moderna similar a otros museos de las capitales europeas y americanas (Mones, 2011, p. 16). El 25 de abril de 1892, Berg renunció a la dirección del museo uruguayo para trasladarse a Buenos Aires con el propósito de asumir la dirección del Museo Nacional de Historia Natural. Por esta razón, el presidente Herrera y Obes designó a Arechavaleta como nuevo director (Mones, 2011, p. 9). A partir de entonces y hasta su muerte en 1912, Arechavaleta fungió como director. ${ }^{15}$

En el Museo se cultivó la botánica como una ciencia útil al Uruguay a través del reconocimiento y aprovechamiento de las especies comerciales y agrícolas poco conocidas que se integrarian a las dinámicas económicas nacional e internacional. Arechavaleta resaltó que el proceso de "aclimatación" de la ciencia en el país se afianzaba a través de la comunidad de naturalistas que se expandía a través de las instituciones patrocinadas por el gobierno, las cuales incorporaban a los jóvenes interesados en desarrollar sus propias actividades científicas.

$A M N M$ no sólo daría a conocer información especializada para la comunidad de naturalistas, sino que aportaría datos de interés para cualquier lector en términos del aprovechamiento económico de varias especies, sobre todo las vegetales. Cabe señalar que la revista presupuso un lector instruido en las generalidades de la historia natural, con recursos suficientes para conseguir los volúmenes y dedicar tiempo a su consulta, además de habilidades para aprovechar el conocimiento científico en su beneficio. Se trata de un estrecho círculo de lectores provenientes de los estratos medio y alto del país.

La institución sufrió un nuevo cambio el 10 de diciembre de 1911, cuando las tres secciones del antiguo Museo Nacional se independizaron para fundar el Museo Nacional de Historia Natural, el Museo Nacional de Bellas Artes y el Museo Nacional Histórico. Esto hizo posible que en un solo museo se concentrara la investigación sobre la naturaleza uruguaya. Tras la muerte de Arechavaleta, el gobierno designó al médico Garibaldi J. Devincenzi (1882-1943) como director (Mones, 2011, p. 13).

En el periodo de esta investigación, el Museo fue parte del creciente entramado institucional de la ciencia uruguaya, el cual se encontraba en el marco de la política económica de los diferentes gobiernos con el objetivo de modernizar los

15 Arechavaleta falleció en 1912. En 1915, su herbario (7.000 plantas) y biblioteca $(1,500$ volúmenes) fueron adquiridos por el gobierno por su importancia científica (Mones, 2011, p. 12). 
rubros productores del país, en especial los basados en las especies vegetales. No hay que dejar de lado que el Museo transitó por un complejo contexto sociopolítico y que estuvo a merced de los vaivenes económicos, aunque la comunidad de naturalistas logró salir avante en el paulatino proceso de desarrollar las actividades científicas de la institución.

\section{La práctica cientifica en las excursiones}

Los naturalistas relacionados con el Museo participaron en varias excursiones con el objetivo de colectar especímenes de la flora del país. Si bien, AMNM no da cuenta de todas las excursiones científicas, es un referente para aproximarse a la práctica botánica in situ, las localidades que los autores recorrieron, y el tipo de observación en campo orientada al reconocimiento de las especies desconocidas por los naturalistas.

Un primer ejemplo se encuentra en "Contribución al conocimiento de los líquenes uruguayos. Lichenes montevidensis" (1894) de José Arechavaleta y J. Müller, escrito que refleja la importancia de las excursiones en el acopio de ejemplares para el Museo y su posterior estudio de gabinete y, en ocasiones, dar a conocer los resultados científicos en $A M N M$. Arechavaleta expuso que "en mis excursiones botánicas" recogió varios ejemplares de líquenes, gracias a lo cual logró "poseer un cierto número", cuyos nombres específicos "me eran desconocidos en su mayor parte" (Arechavaleta \& Müller, 1894, p. 173). Ante la incógnita de su taxonomía, Arechavaleta compartió los especímenes con el Dr. J. Müller, "renombrado liquenógrafo" de Ginebra, quien determinó sus características anatómicas y ubicación taxonómica para publicar los resultados en el folleto titulado Lichenes Montevidensis (Arechavaleta \& Müller, 1894, p. 173). Las excursiones fueron la vía común en que los naturalistas uruguayos y del mundo recababan nuevos especímenes. Los líquenes no son una planta, sino una simbiosis entre especies de hongos, algas y bacterias, aunque en el siglo XIX se les incorporó dentro de la taxonomía vegetal. Dada la falta de conocimiento especializado por Arechavaleta en los líquenes, remitió muestras a su colega suizo, quien determinó las especies a partir de su pericia en la observación anatómica. Esto es un indicio de las redes de colaboración internacional que establecieron los botánicos del Uruguay y Europa occidental a finales del siglo XIX a partir del Museo.

Debido a la carencia de estudios en español sobre la diversidad liquenológicia del Uruguay, Arechavaleta dio a conocer Lichenes Montevidensis en AMNM, por su aporte al conocimiento de cincuenta líquenes uruguayos, los cuales "representan una pequeña parte de los que existen en este país y cuyo hallazgo puede ser fuente de gratas emociones para los que se dediquen a estudiarlos" 
(Arechavaleta \& Müller, 1894, p. 173). La traducción de textos científicos fue una estrategia que Arechavaleta puso en práctica en su papel de editor de la revista para atraer la atención de los naturalistas del país y ganar legitimidad académica al acopiar información extranjera sobre la flora nacional que en el futuro ayudaría a determinar las muestras de líquenes sin recurrir a los extranjeros.

Müller y Arechavaleta (1894) recomendaron a los botánicos uruguayos que efectuaban excursiones por el país que estuvieran atentos a las rocas, las cortezas y las ramas de los árboles, pues era común que alojaran nuevas especies de líquenes "y completamente diferentes de las primeras, y yo, con sumo gusto, las utilizaré para la ciencia" (Arechavaleta \& Müller, 1894, p. 174). El editor hizo un llamado a los lectores a enviar datos y ejemplares de nuevas especies de la flora que consideraran relevantes para incorporarlas al acervo del Museo y estudiarlas detenidamente con el propósito de añadirlas al sistema taxonómico mundial.

Otro ejemplo de la traducción para $A M N M$ es la sintesis del tercer volumen de Revisio generum plantarum (1891-1898) del naturalista alemán Otto Kuntze intitulada "Enumeración de las plantas que recogió el Dr... en esta República" (1899). Arechavaleta indicó que el escrito presentaba interés para la ciencia nacional por lo que tradujo lo referente a las plantas indígenas y así "evitar a otros el enojoso trabajo de compulsar obras voluminosas en busca de unas cuantas especies diseminadas entre mil procedentes de distintas nacionalidades" (Kuntze, 1899, p. 259). AMNM, como otras revistas latinoamericanas, incluyó traducciones del interés de los científicos locales para introducir literatura especializada producida en Europa y Estados Unidos que complementaban su propia práctica científica. Además, refleja que Arechavaleta era conocedor de los estudios científicos sobre la naturaleza uruguaya realizados en el extranjero.

La traducción evidencia que Kuntze (1899) recolectó varios especímenes en su viaje de exploración botánica por Bolivia, Argentina, Paraguay, Brasil y en particular en las orillas del río Santa Lucía y la Sierra de Solís del Uruguay (p. 259). El naturalista alemán determinó en su libro 136 especies uruguayas, de las cuales seis eran nuevas. Los naturalistas locales no eran los únicos que exploraban el país, pues en distintos años hubo exploradores de Europa que recorrían varios países con el propósito de colectar in situ los ejemplares y no depender del envío de sus colegas americanos.

José Arechavaleta (1899) en la primera parte de "Contribución al conocimiento de la Flora Uruguaya" 16 indicó que daba inicio a una serie de escritos sobre

16 Los escritos hemerográficos se publicaron como libro bajo el nombre de Flora uruguaya;
enumeración y descripción breve de las plantas conocidas hasta hoy y de algunas nuevas que 
plantas "nuevas o poco conocidas" conservadas en el herbario del Museo Nacional (p. 273). Acerca de las dicotiledóneas, el autor escribió que hasta entonces "ninguna samidácea de estas regiones uruguayas era conocida" hasta que en 1898 llevó a cabo una excursión a la Cuchilla Negra en el Departamento de Rivera en que colectó dos especies del género Banara, a las que "podemos agregar otra, que desde largo tiempo, conservábamos sin determinar en nuestro herbario particular" (Arechavaleta, 1899, p. 273). Por la novedad de las tres especies de samidáceas, el autor las incluyó en el escrito. La revista fue el medio público en que Arechavaleta circulaba entre sus colegas los resultados de las excursiones y mostraba cómo colaboraba a enriquecer el inventario de la flora nacional. También se aprecia que Arechavaleta en sus excursiones colectaba ejemplares tanto para el Museo como para su colección personal, así como los resultados de ambos acervos contribuyeron a elaborar estudios pormenorizados de las nuevas especies.

Arechavaleta (1903c) en la segunda parte de "Contribución al conocimiento de la Flora Uruguaya" mencionó que Mariano B. Berro había realizado excursiones en los campos altos de Vera, en donde había colectado durante la primavera una pequeña crucífera que "representa un género más en nuestra flora" (p. 2). Esta especie era semejante a otra colectada en la Sierra de la Ventana por el doctor Carlos Spegazzini, aunque se diferenciaba por la presencia de pelos ramificados, silicuas oblongas anchas, no elipticas y estrechas como en la Draba australis (Arechavaleta, 1903c, p. 2). Esta mención evidencia a otros dos naturalistas que efectuaban excursiones en el Uruguay y estuvieron vinculados con el Museo al intercambiar especímenes e información botánica, así como fueron autores en la revista.

En 1903 se tradujo del naturalista francés Joseph-Ernest Gibert el escrito "Flora Uruguaya. Nómina Vernacularia" a partir del libro Enumaeratio plantarum sponte nascentium agro Montevidensi cum synonimis selectis (1873). El libro se compone de una lista alfabética de nombres científicos de plantas uruguayas recogidas por el científico galo. Arechavaleta agregó al escrito original el nombre vulgar en los casos en que hacía falta en la obra original con el objetivo de dar a conocer a los lectores los nombres populares de "los vegetales que viven en la República" y como material de complemento taxonómico para cualquier naturalista uruguayo que se propusiera publicar "un trabajo completo sobre semejante materia" (Gibert, 1903, p. 132). Arechavaleta intervino en varias ocasiones textos de autores extranjeros en otras lenguas para promover su lectura entre el grupo de naturalistas vinculados al Museo. El editor no se

nacen espontáneamente y viven en la República Uruguaya, tomo 1 (1898-1901), tomo 2 (19031905), tomo 3 (1906-1908) y tomo 4 (1909-1911). 
limitó a la traducción o el resumen en $A M N M$, pues en la coproducción añadió datos novedosos o corrigió la información original que consideró equivocada.

El director del Museo indicó que Gibert se había nutrido de los especímenes colectados por Federico Balparda (1839-¿?), ${ }^{17}$ un aficionado a la botánica que habia formado un herbario premiado en la Exposición de Santiago de Chile y después la había regalado al Ateneo de Montevideo; el señor Silva, un farmacéutico que recolectaba plantas medicinales en los alrededores de Montevideo; y el general José María Reyes, autor de Descripción geográfica del territorio de la República O. del Uruguay (1859), naturalistas "apreciados en esta sociedad montevideana" (Gibert, 1903, p. 133). La mención de los colectores muestra la diversidad del grupo de naturalistas uruguayos (militares, sacerdotes, profesionistas, propietarios rurales, burócratas, entre otros), quienes formaban colecciones privadas, donaban ejemplares al Museo e intercambiaban especimenes e información entre sí y con científicos del Viejo Mundo. Estos aprovechaban la información remitida por los uruguayos para publicar sus obras y en ocasiones mencionaban a los colectores locales. Arechavaleta expuso cómo $A M N M$ fue un recurso para el acopio de información proveniente del extranjero y de interés nacional a partir de la traducción y elaboración de resúmenes de los escritos originales.

En el mismo año se publicó "Citharexylum barbinerve Cham. y Schecht. Tendencia hacia la unisexualidad de sus flores" de Arechavaleta. El escrito abordó a una especie de verbenacea indígena, conocida con el nombre popular de taruman. El autor indicó que en diferentes "herborizaciones por la campiña" había observado ejemplares completamente desarrollados a manera de árboles con frutos y sin frutos, lo que manifestaba la bisexualidad, "fenómeno, si así puede llamarse, atrajo nuestra atención y nos propusimos averiguar sus causas" (Arechavaleta, 1903b, p. 150). En las excursiones, Arechavaeta observaba las distintas fases del desarrollo de las especies nativas, las que en ocasiones originaban una investigación particular. Al respecto, Arechavaleta expresó que

en un cultivo de este árbol, hecho por nuestro amigo y compañero de excursiones botánicas, el señor Cantera, constando de un cierto número de ejemplares bien desarrollados, observamos que los unos daban fruto y los otros no, y eso aunque todos se cubrian de flores, eran de la misma generación $y$ se encontraban a cortas distancias. Señalados intencionalmente, averiguamos que se condujeron de igual manera durante dos estaciones consecutivas. Nos encontrábamos, pues, en presencia de un hecho constante. El examen de las flores reveló su verdadera causa, como se verá en la exposición que juzgamos necesaria para la averiguación de este caso, a nuestro juicio, más frecuente de lo que puede creerse, en muchas especies fanerógamas bisexuadas, y que

17 Fue uno de los fundadores de la Asociación Rural de Uruguay en 1870 y promotor de la enseñanza agrícola en el país. 
constituye, un paso más adelantado hacia la unisexualidad o separación de sexos, que el alcanzado por la dicogamia o sea la no simultaneidad de desenvolvimiento de estambres y pistilos en una misma flor, aun cuando sea lo mismo para conseguir el cruzamiento a que tiende la vida vegetal (Arechavaleta, 1903b, p. 150).

La práctica de la observación de los ejemplares por un largo tiempo en su ambiente fue una estrategia para investigar la flora uruguaya. Esto evidencia que Arechavaleta y sus acompañantes exploraban tanto localidades nuevas como conocidas en distintos momentos del año. La observación general de un árbol o una hierba se complementaba con la observación minuciosa como el caso de las flores de la verbena, la que tal vez se realizó in situ con una lupa o tal vez al microscopio en el Museo. Ambos tipos de observaciones permitieron a Arechavaleta analizar la posible transición de la bisexualidad a la unisexualidad en Citharexylum barbinerve. La mención al señor Cantera, otro colector, revela que en torno al Museo hubo diversos individuos interesados en la botánica hasta ahora desconocidos en la historiografia de la ciencia uruguaya.

En la segunda serie de los $A M N M$ se publicó "Apuntes botánicos" (1925a) fechado en 1905, de la autoría de Arechavaleta en que expuso cómo había colectado una nueva especie del género Clematis en una excursión por las serranías de Puntas de Pan de Azúcar. La especie habitaba la zona de peñascos y arroyos, "al examinarla llamó nuestra atención hasta el punto de pensar en nueva especie. En tal creencia arrancamos varios trozos, de los cuales tuvimos la suerte de ver retoñar dos en la primavera siguiente" en las inmediaciones del Museo que resultaron del sexo masculino (Arechavaleta, 1925a, p. 18). En las excursiones no sólo se acopiaban muestras inertes de diferentes plantas para conformar el herbario, sino que en ocasiones fue posible reproducir algunas especies en una pequeña área arbolada del Museo en la cual fructificaron varias plantas para después efectuar su examen taxonómico de gabinete.

Otro escrito póstumo de Arechavaleta publicado en 1925 versa sobre el estudio de nuevas especies de cactáceas, con fecha de 1905. Esta vez se trata de Opuntia monacantha, incluida en el segundo tomo de Flora Uruguaya (1903-1905), en cuya descripción original se consideró que carecía de aguijones a partir de una muestra procedente de Tacuarembó y se incluyó un grabado fotográfico (Arechavaleta, 1925c, p. 41). Tiempo después, el autor continuó estudiando nuevos ejemplares colectados e incluso "pudimos observarlas en su medio natural, de una manera completa, es decir, siguiendo su desarrollo hasta la maduración de sus frutos. De este examen adquirimos la certeza que se trata de dos especies bien distintas" (Arechavaleta, 1925c, p. 41). Una especie correspondía a la lámina incluida en Flora Uruguaya y otra era la observada in 
situ, y ambas diferían del O. monacantha "según lo pudimos comprobar por comparación de ejemplares vivos" (Arechavaleta, 1925c, p. 42). Las excursiones fueron una práctica científica indispensable a la hora de comparar las muestras del herbario con los especímenes vivos en cuanto a su anatomía, color, textura y tamaño, elementos que a Arechavaleta permitieron reflexionar sobre su estudio taxonómico inicial para luego cambiarlo hacia la determinación de tres especies. Las excursiones constituyeron la vía en que los naturalistas uruguayos, como Arechavaleta, contrastaban y corregian los estudios anteriores de su propia autoría y otros científicos para ampliar el inventario de la flora del país, mientras que $A M N M$ fue el medio para comunicar los resultados. La experiencia en el medio en que crecian las especies uruguayas hizo posible que los naturalistas estuvieran atentos a los cambios en el desarrollo de algunas plantas y a partir de ello generar preguntas de investigación, cuyas respuestas publicaron en $A M N M$.

\section{La práctica cientifica en el trabajo de gabinete}

El trabajo al interior del Museo con los especímenes después de colectados en las excursiones fue llevado a cabo con fines de determinación taxonómica y descripción anatómica, así como ampliar el inventario de la naturaleza uruguaya y nutrir la exhibición de ésta. Los escritos que hacen referencia al trabajo de gabinete fueron publicados por Arechavaleta.

En "Contribución al conocimiento de los líquenes...", Arechavaleta (1894) resumió la serie de cartas intercambiadas con el Dr. Müller de Ginebra que acompañaron el envío de 43 líquenes, de los cuales, diez eran especies nuevas. El profesor suizo estaba satisfecho de incorporar nuevos ejemplares a su colección privada de tipos y lamentó que las muestras de algunos líquenes se hubieran maltratado en el trayecto de Montevideo a Ginebra, por lo cual "he notado trazas de otras especies, que no he podido estudiar, por falta de material suficiente, sucede a veces, que es preciso sacrificar varios frutos para hallar esporos en buen estado, de manera que no es posible hacer el examen completo" (Arechavaleta \& Müller, 1894, p. 174). El intercambio de especímenes entre ambos naturalistas resultó fecundo en la determinación de nuevas especies, aunque la fragilidad de los líquenes en el trayecto en barco y ferrocarril entre ambas ciudades implicó la pérdida de cierto material botánico.

En la última carta, Müller solicitó a Arechavaleta "recibir muestras más complejas, de las especies representadas por pequeños fragmentos" con el propósito de concluir el estudio de los líquenes dañados (Arechavaleta \& Müller, 1894, p. 174). La coproducción de conocimiento botánico se produjo en el trabajo de gabinete en Ginebra, aunque no es claro si en una institución suiza o en casa de Müller, y posteriormente en el Museo montevideano se efectuó la 
tarea de incluir las muestras duplicadas en el herbario nacional y las vitrinas de exhibición pública.

José Arechavaleta (1899) en "Contribución al conocimiento de la Flora Uruguaya" incluyó el apartado "Una planta nueva de la flora uruguaya" relativa a la experiencia del botánico Carlos Spegazzini, quien el 17 de mayo de 1898 había recibido de parte de Arechavaleta un "paquetito de plantas uruguayas" para que efectuara la determinación taxonómica mediante la comparación con el material de su herbario personal "a causa de que los ejemplares recogidos eran incompletos, careciendo en su mayoría de flores y frutos" (1899, p. 273). Spegazzini radicaba en La Plata, Argentina, en donde poseía una amplia colección botánica con la cual intercambiaba ejemplares con distintos naturalistas del mundo. El director del Museo aprovechó su cercanía con el botánico italiano para que colaborara en la clasificación taxonómica de algunos especimenes.

A Spegazzini le llamó la atención una rama recolectada en los montes de la sierra de Pan de Azúcar, cuyas hojas enteras y apergaminadas llevaban al pie un par de enormes estípulas, dándole un aspecto semejante al de una Bixacea. Como la primera muestra estaba incompleta, el botánico italiano solicitó a Arechavaleta nuevos ejemplares "más completos y desarrollados" (Arechavaleta, 1899, p. 274). El 14 septiembre, Arechavaleta envió a Spegazzini nuevas muestras en plena floración. El estudio de las flores reveló al botánico de La Plata que se trataba de un género nuevo de samidácea, pues las características anatómicas de la planta no eran semejantes a los géneros clasificados en los tres volúmenes de Genera Plantarum ad exemplaria imprimis in herbariis Kewensibus (1862-1883) de George Bentham y Joseph Dalton Hooker. Spegazzini solicitó a Arechavaleta que obtuviera frutos de la planta para "estar más seguro de la determinación y en tal caso poder dar una descripción completa del nuevo representante de la flora de la Banda Oriental" (Arechavaleta, 1899, p. 274). La práctica de gabinete requería del estudio profundo de las inflorescencias y frutos de las especies fanerógamas para determinar su ubicación en la taxonomía, sobre todo si se sospechaba que era una nueva especie, lo que en ocasiones requería de colectar muestras en distintas épocas del año a partir del reconocimiento del ciclo vital de la especie. La determinación de las nuevas especies requirió de la práctica de colecta de Arechavaleta sumada a la práctica de gabinete de Spegazzini.

E1 26 de noviembre, Arechavaleta remitió a La Plata el fruto "de la interesante samidácea" colectada en una de sus excursiones por uno de sus acompañantes, del cual no se dejó constancia del nombre. Spegazzini señaló que el estudio:

de todo este material me confirmó en mi opinión primitiva y hoy estoy plenamente convencido que se trata de un género y especie nueva de la 
familia de las Samidáceas, género y especie perfectamente caracterizados, que me permito publicar en esta corta relación, tomándome la libertad de dedicar dicho género a su sabio e infatigable descubridor, como testimonio de gratitud y cariño amistoso para con él (Arechavaleta, 1899, p. 276).

Arechavaleta expuso en la correspondencia resumida en su escrito el complejo proceso de determinación taxonómica de un nuevo género y nueva especie del Uruguay que iniciaba con la colecta en una localidad, continuaba con el examen pormenorizado de su anatomía y concluía con la ubicación de la novedad en la clasificación botánica. Un proceso que involucraba a varios especialistas inmersos en una red amplia de coproducción de conocimiento científico, tanto colectores anónimos como destacados naturalistas.

El director del Museo al inicio de "Flora Uruguaya. Enumeración..." indicó que el primer volumen de su magna obra presentaba alrededor de 435 especies vegetales, 150 géneros y 38 órdenes, de las cuales "todas, menos una que otra, figuran en el herbario del Museo Nacional" (Arechavaleta, 1901, pp. VII-XIII). En el herbario se resguardaban libros a manera de álbum, en que cada hoja de papel fuerte, protegida por otra de papel secante, contenía una muestra de cada especie, "sujeta sólidamente con alfileres o con tiras engomadas, de tal manera que pueden ser examinadas cómodamente, una por una, como si fueran estampas impresas", acompañadas de una etiqueta con datos sobre el nombre de cada especie, la localidad de colecta y la época de su floración (Arechavaleta, 1901, pp. VII-XIII). Esta mención es un atisbo al proceso de conformación de los álbumes botánicos para el resguardo de los especímenes y el amplio trabajo botánico que llevaron a cabo Arechavaleta y otros botánicos del país al interior del Museo.

Arechavaleta en "Nueva contribución al conocimiento de la flora del Uruguay" (1925), texto fechado en 1904, explicó que había avanzado en el "arreglo y clasificación de las plantas" del herbario del Museo, gracias a las excursiones de varios uruguayos que "nos proporcionaron varias plantas interesantes, nuevas las unas, y las otras no halladas hasta hoy en esta República" (Arechavaleta, 1925d, p. 1). En el orden de las Compuestas, presentó ocho nuevas especies, acompañadas por grabados fotográficos. Dos de ellas fueron determinadas por el profesor Ignacio Urban del Museo de Historia Natural de Berlin, encargado de elaborar la Flora Brasiliensis, a quien había remitido numerosos ejemplares en varias ocasiones (Arechavaleta, 1925d, p. 2). Arechavaleta (1925d) también indicó que hizo otro envío a Berlín para el profesor Georg Hieronymus (1845-1921),18 quien detectó tres especies nuevas,

18 Botánico alemán que emigró a la Argentina en 1872 para convertirse en ayudante de Paul Günthe Lorentz. En 1881 publicó el catálogo Plantae diaphoricae florae argentinae. En 1892 fue designado curador del Museo de Historia Natural de Berlín. 
"que bautizó con nombres propios, y era un experto en la flora sudamericana" (p. 2). Las redes de colaboración de Arechavaleta fueron amplias con sus colegas en el Viejo Continente, quienes lo auxiliaron en la clasificación científica de varias especies, asi como a incorporar la flora uruguaya en los círculos académicos europeos.

En "Apuntes botánicos" (1925), el director describió al lector varias especies desconocidas del herbario como parte de los resultados taxonómicos para complementar el orden de las Ranunculáceas de Flora Uruguaya, por lo que presentó

\begin{abstract}
cinco especies más con algunas variedades no conocidas. El ilustrado doctor C. Spegazzini en su reciente trabajo Flora de la provincia de Buenos Aires [1905], enumera y descubre doce, de las cuales resultan cuatro exóticas, si se incluye el Delphinium ajaci, no hallado por él, pero que señaló el doctor C. Berg en la Enumeración de plantas europeas que publicó el año 1877. De las ocho que restan, seis viven aquí igualmente, solo dos, a saber: $R$. bulbos y $R$. cimbalaria no se encuentran entre nosotros. En cambio, poseemos cuatro nuevas, propias de nuestra flora con las variedades interesantes de Anemone decapetala. Comenzaremos por el género Clematis como corresponde, del que contábamos con dos únicas especies (Arechavaleta, 1925a, p. 17).
\end{abstract}

El trabajo de gabinete que efectuó Arechavaleta estuvo apoyado con bibliografia especializada de naturalistas con los que había trabajado antes, gracias a lo cual complementó el inventario del Museo y Flora Uruguaya. Entre los especialistas de la flora sudamericana de ambos continentes es claro el vínculo de coproducción de conocimiento botánico de Arechavaleta con Berg, Kuntz, Spegazzini y Müller, relación intelectual expuesta en $A M N M$.

En cuanto al trabajo taxonómico, tres escritos muestran la publicación de nuevas especies de la flora uruguaya. En primer lugar, se tradujo un texto del capitán Baker intitulado "Monocotiledóneas nuevas del Uruguay" (1899), publicado originalmente en Bulletin of Miscellaneous Information, revista de los Reales Jardines Botánicos de Kew, Gran Bretaña, en el número 147 de septiembre 1898. La traducción dio a conocer cinco especies nuevas, una de ellas Zephyranthes longipes, crecía en las orillas arenosas del Río Santa Lucía, Uruguay, y florecía en diciembre (Baker, 1899, p. 287). Este es un ejemplo de las descripciones anatómicas publicadas en el extranjero bajo un lenguaje especializado sobre cada parte de la planta.

El segundo ejemplo es la traducción “Géneros Gynerium y Cortaderia” (1899) de Édouard-François André, escrito original del número de febrero de la Revue Horticole, órgano de la Société Nationale d'Horticulture de France. André abordó 
el género Gynerium con motivo de la reforma taxonómica efectuada por el botánico austriaco Otto Stapf (1857-1933) en el segundo volumen de Gardeners Chronicle de 1897. Arechavaleta tradujo el texto porque en dicho género "se encuentra una planta tan conocida entre nosotros como el Gynerium argenteum (paja brava), parécenos oportuno traducir el trabajo del señor André, para dar a conocer el nuevo nombre que le impone el señor Stapf" (André, 1899, p. 198). $A M N M$ dio cabida a las traducciones de textos extranjeros que atraerian la atención de los naturalistas uruguayos y tal vez de otras naciones sudamericanas que compartian especies vegetales. En el Uruguay se incorporaron estudios europeos que complementaron la coproducción sobre la naturaleza por parte de los científicos locales.

Stapf propuso la creación del género Cortaderia a partir de la revisión pormenorizada del género Gynerium, porque halló diferencias entre $G$. saccharoides, "especie sobre la cual fundaron el género Humboldt y Bonpland," y G. argenteum de Theodor Nees. El botánico austriaco redujo las especies del género Gynerium a solo G. saccharoides, mientras que el resto de especies conformaría el nuevo género Cortaderia "(nombre local que hace alusión a las hojas cortantes de estas plantas) a cuya cabeza se halla" el antiguo G. argenteum (paja brava) (André, 1899, p. 198). El nuevo género en cuestión se hallaría constituído por las especies Cortaderia argentea, "habita el Paraguay, Uruguay, República Argentina y Brasil”, C. araucana, C. speciosa, C. rudiuscula y C. quila (André, 1899, p. 199). Arechavaleta al final de la traducción agregó que había detectado una tercer especie en el campo uruguayo, conocida popularmente como paja brava crespa, a la que otorgó el nombre G. parviflorum, semejante a unos especímenes "que vi en el Herbario de Berlín y que pertenece" al $G$. saccharoides (André, 1899, p. 199). Arechavaleta complementó la traducción a partir de su experiencia en el campo y contrastar la práctica de gabinete del Museo berlinés, con los ejemplares del Museo uruguayo.

El tercer ejemplo es "Vegetación uruguaya. Varias especies nuevas" de Arechavaleta y Guillermo Herter, escrito fechado en 1911 y publicado 1925 sobre la descripción de Spigelia intermedia, la cual crecía en parajes húmedos a las orillas de los pantanos y rios (Arechavaleta \& Herter, 1925, p. 60). Ambos naturalistas aportaron la descripción científica de más de cuarenta especies nuevas a la usanza de la taxonomía de inicios del siglo XX.

\section{La práctica experimental}

La práctica de gabinete en ocasiones estuvo acompañada de la práctica experimental en laboratorios de otras instituciones uruguayas con las que colaboraban los naturalistas del Museo, asi como los laboratorios personales que algunos científicos tenían en sus casas o en las boticas. Un primer ejemplo 
es "Las gramíneas uruguayas" de Arechavaleta (1894a), en que señaló que todo botánico debía reconocer que cada localidad del mundo estaba constituido por un tipo particular de suelo en función de su composición química y rocosa, por lo que no todos eran adecuados "a la primera semilla que deseamos sembrar" (p. 29). El autor recomendó que el botánico y los productores agropecuarios se familiarizaran con algunos experimentos generales para determinar la composición química del suelo en relación con los elementos químicos presentes en cada especie vegetal y, por tanto, relacionados con su utilidad terapéutica, alimenticia, forrajera, textil, tintórea, entre otras.

El director del Museo indicó que después de algunos análisis químicos, determinó que el suelo uruguayo se caracterizaba por contener ácido fosfórico, cal, magnesia y sales de potasio. Esta composición química era propicia para las gramíneas, "base principal de los ricos pastos que nutren y engordan a los animales domésticos que lo pueblan, fuente primera de su prosperidad y riqueza" (Arechavaleta, 1894a, p. 30). Las gramíneas eran el cimiento de la producción agricola, ganadera y peletera del país, así como el elemento que promovía el comercio con Europa occidental, por lo que su estudio científico resultaba de la mayor importancia para el gobierno, la sociedad y los naturalistas.

En 1903, José Arechavaleta publicó una carta dirigida a Carlos Regúnaga, jefe del Laboratorio Municipal de Química, fechada el 21 de julio de 1897. La comunicación tuvo como propósito remitir algunas gramíneas descritas en la Agrostología Uruguaya para conocer su composición y valor alimenticio como elemento forrajero, pues era un estudio "útil a los intereses generales del Estado todo lo que tenga relación con los progresos agronómicos" (Arechavaleta, 1903a, p. 103). En esta carta, Arechavaleta continuó su proyecto de efectuar análisis químicos de diferentes especies uruguayas para comprobar su valor como materia prima para diferentes actividades económicas. Como el Museo carecía de un laboratorio especializado, solicitó apoyo a otra institución del país y a un científico con quien mantenía buenas relaciones académicas y personales.

Carlos Regúnaga elaboró el escrito "Gramíneas uruguayas. Tercera parte. Agrostología aplicada" (1903) como respuesta a la petición de Arechavaleta, el cual incluyó un apartado sobre los análisis químicos de algunas gramíneas, con énfasis en las especies útiles para la formación de prados destinados a apacentar ganado y cultivos de consumo humano. Regúnaga (1903) explicó que los resultados de los estudios botánicos en ocasiones requerian de efectuar experimentación, práctica entendida como el "estudio detenido que conduce al conocimiento perfecto de las condiciones propias de cada especie” (p. 101). El autor presentó el análisis químico de las gramíneas forrajeras "encontradas en los parajes visitados. Poseemos otras recogidas después con el propósito de 
analizarlas también, a fin de contar con el mayor número posible, para llegar a conocer las más importantes como elemento forrajero" (Regúnaga, 1903, p. 102). El escrito de Regúnaga revela que el estudio de gabinete a partir de las especies colectadas se prolongó hacia la experimentación para sancionar la utilidad de éstas, pues los análisis químicos eran costosos y era inviable realizados con todos y cada uno de los ejemplares del herbario del Museo.

Regúnaga (1903) recomendó a Arechavaletta que seleccionara las leguminosas uruguayas más destacadas a su juicio para que en el futuro se llevaran a cabo análisis químicos con el objetivo de determinar la composición con la mayor cantidad de "elementos azoados, que en la alimentación de los animales herbivoros juegan un rol semejante al que desempeña la carne en la del hombre" (p. 102). Los análisis químicos estuvieron orientados a sancionar la utilidad de ciertas especies como base de las principales actividades económicas del país. El vínculo entre el Laboratorio Municipal y el Museo hizo posible coproducir la caracterización científica de algunas especies útiles y hacer públicos los resultados en $A M N M$ para que estuvieran al alcance de los grupos económicos y políticos.

Arechavaleta (1925b) en "Breves apuntes sobre algunas gramineas de propiedades tóxicas para los herbivoros", texto escrito en 1905, incluyó un extracto sobre el informe relativo a la vizcachera (Stipa leptostachya), sometida al "método general de investigaciones para la extracción de los alcaloides", cuyo resultado fue la detección de una sustancia tóxica atribuible a un glucoside análogo al ácido cianhídrico (p. 56). Dada la toxicidad de la vizcachera para el ganado, el autor emprendió un análisis químico para determinar el grado de afectación que podría producir en los animales y hacer un llamado a los ganaderos para que eliminaran la planta de sus propiedades rústicas.

Arechavalera (1925b) concluyó su exposición sobre los resultados experimentales referentes a Stipa leptostachya con presuntas propiedades tóxicas “impulsándonos a ponerlas en conocimiento de los lectores" (p. 57). La revista del Museo cumplió la función de comunicar los resultados científicos producidos por los naturalistas para beneficio de la sociedad, ya fuera con fines económicos, comerciales, terapéuticos y de salud pública. El autor explicó que había tenido noticia de un caso de los campos de Arazatí, en que

un animal vacuno entraba a pacer en un cierto radio, bien señalado por sus efectos, no tardaba en presentar señales de envenenamiento: agitación, hinchazón y muerte breve. En presencia de estos casos, frecuentemente repetidos, fuimos consultados sobre el particular y examinamos varias plantas de aquel lugar (...) Aparte de este caso, tenemos otros análogos, que nos indujeron a sospechar varias plantas, de cuyo examen químico obtuvimos resultados negativos, igual al que nos dieron otros cinco o seis análisis hechos más tarde con el mismo propósito. Para alcanzar la verdad en estos asuntos de observación práctica, era preciso estar en otras condiciones. Reducidos al examen de plantas que nos remitian por

\section{anuario.}


sospechosas, se corría el riesgo de completa esterilidad, a no dar por casualidad con la especie tóxica. Cosa distinta se obtendría si los que se dedican a la ganaderia poseyeran nociones sobre las plantas vulgares que tienen continuamente ante la vista y supieran distinguir, cuando menos, una col de una lechuga, como vulgarmente se dice. Pero no sucede así, desgraciadamente, de manera que no se llegará al descubrimiento de esos vegetales nocivos si no muy tarde, o por mera casualidad (Arechavaleta, 1925b, p. 47).

La cita indica cómo Arechavaleta fue reconocido por los ganaderos como un especialista en botánica que podría ayudarlos a resolver una problemática que enfrentaba este grupo económico. La consulta por los ganaderos hizo que el director del Museo se trasladara a Arazatí a observar y colectar diferentes plantas con el propósito de someterlas al análisis químico en Montevideo. La práctica in situ fue complementada con la práctica experimental para solucionar diferentes preguntas que afectaban a la economía del país. La posterior remisión de ejemplares al Museo por parte de los ganaderos indica el valor que este grupo económico tuvo hacia la institución. Resalta el comentario del autor sobre la importancia de que la población uruguaya estuviera instruida en cuestiones botánicas generales para que fungieran como colectores de especímenes desconocidos para el Museo.

\section{La utilidad de la botánica}

$A M N M$ promovió en varios de sus escritos botánicos la utilidad de las especies uruguayas en distintos rubros económicos y terapéuticos, ya fuera la sanción académica de los usos populares de las especies o la recomendación de usos desconocidos a partir del análisis químico. Un primer ejemplo se encuentra en "Las gramíneas uruguayas" de José Arechavaleta (1894a) en que señaló que de todas las familias vegetales, las gramíneas presentaban el mayor número de especies útiles al ser humano, por ejemplo, el trigo, la cebada, el arroz, la caña de azúcar y el maíz (p. 29). No obstante, la mayoría del aprovechamiento agrícola de dichas plantas en el Uruguay aún no alcanzaba una modernización completa a pesar de las distintas politicas públicas de los gobiernos y las acciones de las agrupaciones económicas. Para el naturalista, el Uruguay debía modernizar su agricultura, pues los "pueblos más prósperos" eran los que con mayor afán se dedicaban al cultivo de toda clase de especies, como apreciaban los viajeros sudamericanos en Francia, Bélgica y Alemania (Arechavaleta, 1894a, p. 29). Arechavaleta mostró al lector el referente científico y económico de países de Europa occidental para reproducir las experiencias exitosas en el campo uruguayo, pues la ciencia había intensificado la producción agrícola en dichas 
naciones con amplia repercusión económica.

Arechavaleta (1894a) centró sus estudios en las gramíneas por el señalado predominio que presentaban sobre otras especies vegetales en el Uruguay y por el papel que "desempeñan en su economía general, me propuse dedicar preferente cuidado a fin de hacerlas conocer más tarde para que puedan aprovecharse en la formación de prados” (p. 30). Aquí es explícito cómo la práctica de Arechavaleta se orientó hacia la flora útil desde el conocimiento científico, incluyendo dar a conocer sus resultados en la revista del Museo. Para Arechavaleta, los forrajes eran

la base de toda agricultura racional (...) si quieres trigo haz prados; o mejor, quien posee pastos tiene pan. Por eso vemos que en los países más adelantados se aumenta cada día la superficie territorial consagrada a los prados; y aunque la indole de nuestro trabajo no nos permite tratar esta cuestión con la amplitud que merece, no dejamos por eso de mencionar especialmente las gramineas que a nuestro juicio poseen mejores cualidades alimenticias, y una vez establecidos los caracteres que las distinguen, señalaremos el terreno que más les conviene y la época de su desarrollo. Si conseguimos dar a conocer las especies forrajeras más importantes y útiles del Uruguay, habremos alcanzado el objeto principal que nos propusimos al emprender este trabajo, en el que hemos perseverado durante 30 largos años por inclinación natural y el amor que profesamos a la ciencia (Arechavaleta, 1894a, pp. 30-31).

El naturalista deja ver que uno de los objetivos de su escrito era convencer al lector de la importancia de ampliar los prados para el ganado, uno de los rubros económicos en expansión en el país. Desde el ámbito de la ciencia y del órgano público del Museo, Arechavaleta esgrimió argumentos para ampliar la frontera agrícola en detrimento de la vegetación "inutil", la cual era de interés científico para el inventario de la flora nacional, pero carecía de valor económico. El director del Museo deja ver que las características de la especie de gramínea por sí misma no ayudaba al agricultor para aprovechar al máximo el rendimiento del cultivo, se requería de los conocimientos edafológicos y reconocer las fases de su desarrollo biológico. El autor hizo un llamado a los naturalistas del país para que "aquellos que posean algún conocimiento de esta familia de plantas, sabedores de la dificultad que presenta por la uniformidad de sus órganos vegetativos" comunicaran sus conocimientos (Arechavaleta, 1894a, p. 31). La ciencia requería de compartir los resultados entre otros individuos para alcanzar el bien común. Arechavaleta en varios escritos concluyó con un apartado sobre la importancia de difundir las especies útiles entre el público, una tarea científica que abarcó gran parte de su vida.

En 1899, Mariano B. Berro en "La vegetación uruguaya. Plantas que se hacen distinguir por alguna propiedad útil o perjudicial" señaló que daba a conocer su estudio por el "servicio que puede prestar la publicidad" de la flora nacional a 
partir de descripciones de "interesantes vegetales que viven en nuestros campos, montes y aguas" (Berro, 1899, p. 93). El autor también se propuso "aumentar entre mis compatriotas la afición a los estudios botánicos tan útiles como agradables" (Berro, 1899, p. 93). De nuevo se aprecia el propósito de los naturalistas vinculados con el Museo de comunicar sus resultados científicos sobre la flora uruguaya útil al país con el público, ya fueran otros naturalistas o los grupos económicos y políticos. De igual manera, Berro mostró el propósito de que otros connacionales se sumaran a la práctica botánica porque era una disciplina sencilla para iniciarse como aficionado y útil al individuo y la sociedad.

Acerca de las especies vinculadas con el rubro ganadero, el autor abarcó algunos hongos, como Puccinia graminis, conocida como polvillo, el cual crecía sobre las plantas de trigo bajo la forma de un polvo rojizo en octubre y noviembre. Berro (1899) incluyó su descripción para alertar a los dueños de ganado acerca de que si las vacas comian forraje conteniendo polvillo en ocasiones sufría daño en la salud (p. 95). Otra especie fue Cyperus riflexus o negrillo, especie que convivia con las gramíneas por lo que eran pacidas por los ganados, "ayudando a su alimentación aunque son mucho más pobres en principios nutritivos" (Berro, 1899, p. 106). Sobre el Equisetum ramosissimum o cola de caballo, Berro (1899) indicó que el ganado vacuno, cuando pacía esta planta, lo dañaba, causándole diarrea sanguinolienta, incluso "casos de aborto y enflaquecimiento" (p. 98). Un aspecto útil de $A M N M$ fue exponer advertencias sobre las implicaciones nocivas o positivas de su ingesta por el ganado y así alertar a los ganaderos de la presencia de algunos hongos o helechos en sus propiedades rústicas.

Las plantas de utilidad para las actividades artesanales que Berro (1899) resaltó fueron Bovista sp. o esponja, abundante en los campos, al madurar por su textura se parecía a las esponjas de mar, se usaba para mechas de candiles; Tillansia usneoides o barbas del monte, planta epífita que servía para rellenar pieles de animales, colchones y almohadas, así como para envolver objetos frágiles en los transportes; y Tillancia macrocnemis, de cuyas hojas se extraía una fibra textil para jarciería (pp. 97-101). El autor dejó constancia del uso económico de algunas especies para distintos rubros artesanales de consumo popular que requería de mayor estudio científico para incorporarlas como materias primas a la industria.

"Flora Uruguaya" (1905a) también destacó algunas especies medicinales. Arechavaleta mencionó Hamamelis virginica y Heuchera americana por su valor astringente para gargarismos y lociones; las grosellas Saxifraga crassifolia y Hydrangea Thunbergii, usadas para infusiones; y del Liquidamar styracifolia se obtenía un bálsamo para los boticarios (Arechavaleta, 1905a, p. XXXVIII). La 
búsqueda de plantas terapéuticas, ya fueran de uso popular o de recién hallazgo, fue constante en los escritos de $A M N M$ dentro de la dinámica naturalista de la época.

En un sentido similar, José Arechavaleta (1906) en la continuación de "Flora Uruguaya" señaló nuevas especies que presentaban aplicaciones terapéuticas, por ejemplo, Eupatorium triplinerve, útil en infusiones digestivas y E. perfoliatum era diurético, sudorífico y emético. También se reconocieron las propiedades terapéuticas de Mikania scandens o guaco "abundante en nuestros campos y selvas"; Ageratum conyzoides "originario de esta región" se usaba como febrífugo y para el tratamiento de pneumatosis del tubo digestivo; y Buccharis ochracea, $B$. articulata y $B$. genistelloides, conocidos como carqueja, se usaban en los pueblos como infusión para combatir indigestiones (Arechavaleta, 1906, p. 1). El director del Museo expuso en sus escritos información sobre los usos populares de varias plantas, las creencias sobre sus efectos y recomendó que la sanción científica las comprobara o refutara. Los naturalistas, igual que los médicos y farmacéuticos, aprovechaban el conocimiento popular sobre la flora para llevar a cabo sus propias investigaciones bajo la metodología positiva.

\section{Conclusiones}

El Museo antes de 1890 tuvo una vida institucional complicada por el contexto político, social y económico del Uruguay. A partir de la presidencia de Herrea y Obes, el Museo contó con recursos gubernamentales para emprender su modernización con el apoyo de la comunidad de naturalistas del país, que si bien era reducida, llevó a cabo varios proyectos científicos en su interior. Los naturalistas uruguayos encontraron en el Museo un espacio para la investigación científica a la altura de otros países europeos y americanos, así como dieron a conocer sus resultados científicos en $A M N M$, revista similar a los órganos impresos de los museos del mundo.

La revista fue un espacio académico que formalizó las redes intelectuales de los naturalistas uruguayos con sus pares en Europa y América al presentar traducciones y correspondencia de utilidad para los lectores en relación con la flora del país. Estos vínculos databan de tiempo atrás entre los naturalistas de ambos continentes, pero el Museo hizo posible formalizarlas mediante la modernización de la institución museística uruguaya.

La coproducción de conocimiento botánico por parte de los naturalistas radicados en el Uruguay y el extranjero en la investigación de la naturaleza nacional es palpable en $A M N M$, ya fuera en las traducciones de escritos que aportaban información sobre las especies uruguayas o en los escritos producidos en coautoría por Arechavaleta con Müller, Herter y Spegazzini, como resultado de la circulación de los especímenes entre ambos continentes. 
Como parte de la coproducción de conocimiento, AMNM también evidencia el constante envío de ejemplares entre los naturalistas del Museo y sus colegas con el propósito de dar a conocer los ejemplares colectados e intercambiar opiniones especializadas para ampliar el inventario de las especies uruguayas y su utilidad económica. Los ejemplares nacionales circularon por varios países, cuestión que será posible profundizar con fuentes de archivo cuando sea posible consultarlas.

Las excursiones fueron el principal medio de acopio de ejemplares de la flora uruguaya para el Museo, los botánicos residentes en el país y los naturalistas extranjeros de Europa y Argentina. La práctica de la colecta in situ descrita en $A M N M$ ejemplifica las actividades del grupo de naturalistas vinculados con el Museo como parte de la institución o aficionados que salian a recorrer el país. Estos naturalistas uruguayos observaban, colectaban y herborizaban en cada excursión, para luego analizar los ejemplares en la práctica de gabinete para acrecentar el acervo del Museo o una colección privada.

La práctica de gabinete de los autores analizados en esta investigación tuvo como base el estudio anatómico de los ejemplares, la clasificación y determinación taxonómica, la inclusión de los ejemplares en el catálogo del Museo y de otras instancias extranjeras, el resguardo y en ocasiones exhibición para el público, y la escritura y publicación de escritos científicos en AMNM. Arechavaleta, André, Regúnaga, Gibert y Berro se interesaron en sumar sus estudios a la discusión botánica internacional a partir de $A M N M$, siguiendo los parámetros de la escritura académica europea.

Algunos escritos refieren la práctica de laboratorio en el estudio químico de ciertos ejemplares con el propósito de determinar su utilidad terapéutica, alimenticia, tintórea, etcétera, a partir de los vínculos entre el Museo y el Laboratorio Municipal, pues fueron las instituciones en que laboraba la comunidad de naturalistas uruguayos.

La mayoría de los escritos botánicos de $A M N M$ estuvieron orientados en hacer pública la utilidad de las especies vegetales del país a partir de la sanción científica, no sólo desde la perspectiva de la cultura popular. En este sentido, los estudios botánicos abordaron tanto las especies alimenticias, terapéuticas, industriales, artesanales, comerciales y forrajeras como las especies nocivas, plagas y dañinas al ganado. Se trata de una perspectiva utilitaria de la botánica, en que los naturalistas de $A M N M$ no sólo efectuaron estudios meramente taxonómicos, sino que en todo momento buscaron la utilidad de cada especie para comunicarla al público, ya fueran otros naturalistas del Uruguay y el extranjero, ganaderos, terratenientes, industriales, médicos, boticarios y políticos. 
La revista hizo posible que el Museo ganara una paulatina legitimidad académica al interior de la comunidad científica uruguaya al agrupar a los naturalistas del país, así como visibilizar a la institución y a la comunidad en el extranjero, sobre todo con los científicos de Europa y Argentina, con quienes se estableció un diálogo académico, gracias a la correspondencia institucional, el canje de ejemplares de la revista y los viajeros que recorrian el país. Al mismo tiempo, también se construyó una legitimidad politica con los gobernantes en turno y las élites económicas mediante los resultados de investigación botánica orientados a contribuir a la modernización de la agricultura, la silvicultura y la ganadería a partir de la sanción de la utilidad de las especies vegetales, ya fuera de larga tradición o desconocidas en sus aspectos económicos.

AMNM presenta otros temas científicos sobre la fauna uruguaya, los recursos minerales, la composición geológica del territorio y aspectos arqueológicos y antropológicos. Estos temas tampoco han sido analizados dentro de la dinámica científica de la revista ni del Museo. Por último, la fuente de archivo relativa a las actividades editoriales de Arechavaleta y la correspondencia institucional del Museo con los naturalistas extranjeros y nacionales complementará esta investigación, una vez que la pandemia de la covid-19 permita la consulta del archivo.

\section{Bibliografia}

Aguilar, H. A. (2009). Carlos Berg: pasen señores y vean... El Carnotaurus. Boletín del Museo Argentino de Ciencias Naturales Bernardino Rivadavia 10(104).

André, É.-F. (1899). Géneros Ginerium y Cortasderia. Anales del Museo Nacional de Montevideo 2.

André, F. \& De Courtois, S. (2001). Edouard André, 1840-1911: Un paysagiste botaniste sur les chemins du monde. Besanc,on: Ed. de 1'Imprimeur.

Arechavaleta, J. (1894a). Las gramíneas uruguayas. Anales del Museo Nacional de Montevideo 1.

Arechavaleta, J. (1894b). Personal del Museo. Anales del Museo Nacional de Montevideo 1.

Arechavaleta, J. \& Müller, J. (1894). Contribución al conocimiento de los líquenes uruguayos. Lichenes montevidensis. Anales del Museo Nacional de Montevideo 1. 
Arechavaleta, J. (1899). Contribución al conocimiento de la Flora Uruguaya. Anales del Museo Nacional de Montevideo 2.

Arechavaleta, J. (1901). Flora Uruguaya. Enumeración y descripción breve de las plantas conocidas hasta hoy $y$ de algunas nuevas que nacen espontáneamente y viven en la República Uruguaya e Informaciones. Anales del Museo Nacional de Montevideo 3.

Arechavaleta, J. (1903a). Carta al señor Carlos Regúnaga, Jefe Director del Laboratorio Municipal de Química. Anales del Museo Nacional de Montevideo 4.

Arechavaleta, J. (1903b). Citharexylum barbinerve Cham. y Schecht. Tendencia hacia la unisexualidad de sus flores. Anales del Museo Nacional de Montevideo 4.

Arechavaleta, J. (1905a). Flora Uruguaya. Anales del Museo Nacional de Montevideo 5.

Arechavaleta, J. (1905b). Prólogo. Anales del Museo Nacional de Montevideo 5.

Arechavaleta, J. (1906). Flora Uruguaya. Anales del Museo Nacional de Montevideo 6.

Arechavaleta, J. (1925a). Apuntes botánicos. Anales del Museo Nacional de Montevideo 1, segunda serie.

Arechavaleta, J. (1925b). Breves apuntes sobre algunas gramíneas de propiedades tóxicas para los herbivoros. Anales del Museo Nacional de Montevideo 1, segunda serie.

Arechavaleta, J. (1925c). Esclarecimiento sobre algunas cactáceas. Anales del Museo Nacional de Montevideo 1, segunda serie.

Arechavaleta, J. (1925d). Nueva contribución al conocimiento de la flora del Uruguay. Anales del Museo Nacional de Montevideo 1, segunda serie.

Arechavaleta, J. \& Herter, G. (1925). Vegetación uruguaya. Varias especies nuevas. Anales del Museo Nacional de Montevideo 1, segunda serie.

Azuela, L. F. (2020). A brief account of german geological research in Mexico and its local collaborators (1824-1847). Earth Sciences History 39(2).

Baldwin, M. (2015). Making Nature. The History of a Scientific Journal. Chicago: The University of Chicago Press. 
Baptista, B. (2005). La temprana vitivinicultura en el Uruguay (1874/1930). ¿Tradición o Innovación? (Tesis de Maestría). Universidad de la República, Montevideo.

Baker. (1899). Monocotiledóneas nuevas del Uruguay. Anales del Museo Nacional de Montevideo 2.

Beretta, A. (2020). Terratenientes, nueva agricultura e inicios de la ciencia en el Uruguay de la modernización (1870-1900). Redes 25(48).

Berro, M. B. (1899). La vegetación uruguaya. Plantas que se hacen distinguir por alguna propiedad útil o perjudicial. Anales del Museo Nacional de Montevideo 2.

Bonfanti, D. (2012). Desde la disconformidad eufórica hasta el pesimismo melancólico. Elites, Estado y técnicos extranjeros en los procesos de innovación agrícola en el Uruguay de los centenarios (1910-1930). En A. Beretta (coord.); Agricultura y modernización, 1840-1930. Montevideo: Universidad de la República.

Castellanos, A. (1973). Breve historia de la ganadería en el Uruguay. Montevideo: Banco de Crédito.

Cheroni, A. (1988). Políticas científico-tecnológicas en el Uruguay del siglo XX. Montevideo: Universidad de la República.

Csiszar, A. (2018). The Scientific Journal. Authorship and the Politics of Knowledge in the Nineteenth Century. Chicago: The University of Chicago Press.

De Greiff, A. \& Nieto, M. (2006). What we still do not know about South-North technoscientific exchange. En R. E. Doel \& T. Söderqvist (eds.); The Historiography of Contemporary Science, Technology, and Medicine. Writing recent science. London: Routledge.

Drayton, R. (2000). Nature's Government: Science, Imperial Britain, and the "Improvement" of the World. Nueva Haven: Yale University Press.

Endersby, J. (2008). Imperial Nature. Joseph Hooker and the Practices of Victorian Science. Chicago: Chicago University Press.

Fernández Saldaña, J. M. (1945). Diccionario uruguayo de biografias. Montevideo: Editorial Amerindia.

Fiedczuk, A. \& De Magistris, A. (2016). Carlos Spegazzini y su impronta en la historia y ecología de Santa Catalina. Revista de Divulgación Técnica Agropecuaria, Agroindustrial y Ambiental Facultad de Ciencias Agrarias 3(1). 
Fox, R. (2016). Science without Frontiers. Cosmopolitanism and National Interests in the World of Learning, 1870-1940. Corvallis: Oregon State University Press.

Gibert, J.-E. (1903). Flora Uruguaya. Nómina Vernacularia. Anales del Museo Nacional de Montevideo 4.

Goicoetxea, A. (1993). Un naturalista vasco en Uruguay: José Arechavaleta. Bilbao: Laga.

Howsam, L. (2006). Old Books and New Histories. An Orientation to Studies in Books and Print Culture. Toronto: University of Toronto Press.

Katinas, L., Gutiérrez, D. G. \& Torres, S. S. (2000). Carlos Spegazzini (18581926): Travels and Botanical Work on Vascular Plants. Annals of the Missouri Botanical Garden 87(2).

Klappenbach, M. A. (2004). Larrañaga y el viejo Museo. Montevideo: Museo Nacional de Historia Natural y Antropología.

Kuntze, O. (1899). Enumeración de las plantas que recogió el Dr... en esta República. Anales del Museo Nacional de Montevideo 2.

Kuntze, O. (1891-1898). Revisio generum plantarum:vascularium omnium atque cellularium multarum secundum leges nomenclaturae internationales cum enumeratione plantarum exoticarum in itinere mundi collectarum. Leipzig: A. Felix, 3 vols.

Mañé, F. (1996). Historia de la ciencia en el Uruguay: Primera mitad del siglo XIX. Montevideo: Universidad de la República.

Mañé, F. (2017). José Arechavaleta (1838-1912). Creador de la investigación en ciencias naturales en el Uruguay. En V. Pellegrino, S. Klimavicius, I.Vique \& G. Varela (comps.); Científicos uruguayos: destacadas contribuciones al conocimiento biológico durante los siglos XIX y XX. Montevideo: Asociación de Profesores de Biología/Consejo de Formación en Educación.

Mones, A. (2011). Apuntes para una historia del Museo Nacional de Historia Natural, Uruguay. Museo Nacional de Historia Nacional (4).

Moraes, M. I. (2012). Las transformaciones rurales en el Uruguay de la segunda mitad del siglo XIX: una sintesis revisada. En A. Beretta (coord.); Agricultura y modernización, 1840-1930. Montevideo: Universidad de la República. 
Nyhart, L. K. (1991). Writing Zoologically: The Zeitschrift für wissenschaftliche Zoologie and the Zoological Community in Late. En P. Dear (ed.); The Literary Structure of Scientific Argument. Philadelphia: University of Pennsylvania Press.

Peluffo, A. (1989). José Arechavaleta (1838-1912). En H. Gutiérrez Blanco (ed.); Médicos uruguayos ejemplares. Montevideo: Sindicato Médico del Uruguay, vol. II.

Portillo, J. (1995). Historia de la medicina estatal en Uruguay (1724-1930). Revista Médica del Uruguay 11.

Raj, K. (2007). Relocating Modern Science. Circulation and the Construction of Knowledge in South Asia and Europe, 1650-1900. London: Palgrave.

Regúnaga, C. (1903). Gramíneas uruguayas. Tercera parte. Agrostología aplicada. Anales del Museo Nacional de Montevideo 4.

Rela, W. (2002). Personalidades de la cultura en el Uruguay. Montevideo: Biblioteca Nacional.

Roberts, L. (2009). Situating Science in Global History. Local Exchanges and Networks of Circulation. Itinerario 33(1).

Sanz, V. (2016). Aportes de la Asociación Rural del Uruguay a la viticultura en Uruguay (1872-1900). Mundo Agrario 1(34).

Scarone, A. (1937). Uruguayos contemporáneos. Montevideo: Casa A. Barreíro y Ramos S. A.

Secord, J. A. (2004). Knowledge in Transit. Isis 95(4).

Shaffer, S., Roberts, L., Raj, K. \& Delbourgo, J. (2009). Introduction. En S. Shaffer, L. Roberts, K. Raj y J. Delbourgo (eds.); The Brokered World. GoBetweens and Global Intelligence, 1770-1820. Sagamore: Watson Publishing International LLC.

Spegazzini, C. (1903). Stipeae platenses. Anales del Museo Nacional de Montevideo 4.

Stafleu, F. A. (1978). Kuntze, Carl Ernst Otto. En C. C. Gillispie (ed.); Dictionary of Scientific Biography. New York: Scribner, vol. XV.

Recibido: 03 de Abril de 2021 